\title{
Post-transplant manifestation of ankylosing spondylitis: a case report and review of literature
}

\author{
Anna Zawiasa-Bryszewska ${ }^{1 *}$ D, Olga Brzezińska², Ilona Kurnatowska ${ }^{1}$ and Joanna Makowska²
}

\begin{abstract}
Background: Ankylosing spondylitis (AS) is an insidiously progressive and debilitating form of arthritis involving the axial skeleton, characterized by chronic back pain and progressive spinal stiffness, and lessening of pain and stiffness with exercise. Due to subsequent manifestation in different organs, AS causes reduction in life expectancy, so early diagnosis and treatment are of great importance. No AS cases have been reported in solid-organ transplant recipients yet.

Case presentation: A 58-year-old woman with end-stage renal disease due to chronic glomerulonephritis, after allogenic kidney transplantation 25 years earlier, with stable, good graft function, treated with chronic immunosuppressive therapy based on cyclosporine A, mycophenolate mofetil, and prednisone, with no previous history of a connective tissue disease presented fever up to $39^{\circ} \mathrm{C}$ accompanied by pain localized in sacroiliac region radiating to the left lower limb. Detailed diagnostic procedures and $\mathrm{x}$-rays of the lumbar spine and of the targeted sacroiliac joints revealed lesions characteristic of AS. Sulphasalazine was added to standard immunosuppression regimen with good clinical results.

Conclusions: We report an adult kidney transplant recipient with a new onset of AS. The risk of relapse or new onset of inflammatory disease in transplant recipients is extremely low due to immunosuppressive therapy following transplantation. However, when it occurs, the clinical presentation is commonly atypical, often leading to delayed diagnosis.
\end{abstract}

Keywords: Ankylosing spondylitis case report, Kidney transplantation, Chronic back pain, Immunosuppressive treatment

\section{Background}

The appearance of inflammatory and autoimmune diseases is quite low in patients after solid organ transplantation [1]. Adequate immunosuppressive therapy usually mitigates the risk of relapse or new onset of an autoimmune disease in this population [2]. The clinical presentation of inflammatory diseases in patients after transplantation is commonly atypical, often leading to delayed diagnosis.

Musculoskeletal pain is a common problem in kidney transplant (KTx) recipients, however, an acute inflammatory arthritis is rare. The differential diagnosis of joint and back pain is broad and includes septic arthritis,

\footnotetext{
*Correspondence: ania_zawiasa@go2.pl

${ }^{1}$ Department of Internal Diseases and Transplant Nephrology, The Medical University of Lodz, Lodz, Poland

Full list of author information is available at the end of the article
}

systemic infection, crystal arthropathies, autoimmune rheumatologic disorders, and medication-related adverse reactions [3].

Ankylosing spondylitis (AS), previously known as Bechterew's disease, is a chronic, progressive inflammatory disease with a diverse clinical presentation [3]. AS is characterized by inflammation and new bone formation leading to fusion of the spine and sacroiliac joints. Chronic back pain, progressive spinal stiffness and improvement of pain and stiffness with exercise are the most common features of the disease [4]. Other musculoskeletal manifestations of AS include arthritis, enthesitis and dactylitis [5]. Clinical symptoms most frequently begin in late adolescence or in young adults, with $80 \%$ before the age of 30 and with a 3:1 to 2:1 male to female ratio $[6,7]$. 
In addition to spinal inflammation, AS is characterized by a broad clinical spectrum of the extra-articular manifestations like uveitis, psoriasis, inflammatory bowel disease, or aortic insufficiency $[8,9]$ as well as an increased cardiovascular risk, several pulmonary, renal, and neurological complications or depression [10].

We present a case of late onset of seronegative spondyloarthropaty in KTX patient despite ongoing immunosuppressive treatment. The following description applies to become unusual both in terms of gender, age, comorbidities of the patient, as well as the fact that the patient was constantly treated with triple immunosuppressive regimen.

\section{Case presentation}

A 58-year-old woman with end-stage renal disease caused by chronic glomerulonephritis, after allogenic KTx 25 years earlier, with stable, good graft function, treated with chronic immunosuppressive therapy based on cyclosporine A (CsA), mycophenolate mofetil (MMF), and prednisone, with no previous history of a connective tissue disease was admitted to the Nephrology Department with fever up to $39^{\circ} \mathrm{C}$ accompanied by pain localized in the sacroiliac region radiating to the left lower limb of 2 weeks duration. Physical examination revealed nothing specific, her vital signs were stable. Initial laboratory data indicated an elevated $\mathrm{C}$-reactive protein (CRP) concentration to $287 \mathrm{mg} / \mathrm{l}$ (normal range $<5 \mathrm{mg}$ / l), white blood count (WBC) was $6.4 \times 10^{3} / \mu \mathrm{l}$, haemoglobin concentration $10.6 \mathrm{~g} / \mathrm{dl}$, serum procalcitonin was negative. Urinalysis showed proteinuria $0.1 \mathrm{~g} / \mathrm{l}$, and 15 to 20 leukocytes /higher-power field (HPF), with no erythrocytes in urinary sediment. The graft function was stable (serum creatinine concentration $(\mathrm{sCr}) 95 \mu \mathrm{mol} / \mathrm{l}$ with estimated glomerular filtration rate $\left(\mathrm{eGFR}_{\mathrm{CKD}-\mathrm{EPI}}\right)$ $\left.57.2 \mathrm{ml} / \mathrm{min} / 1.73 \mathrm{~m}^{2}\right)$, serum uric acid was $7.8 \mathrm{mg} / \mathrm{dl}$ (normal range 4.2-6.8 $\mathrm{mg} / \mathrm{dl}$ ), alanine and aspartate aminotransferase as well as bilirubin concentration were within normal limits. The blood level of CsA was in the range appropriate to the period after KTx. Hepatitis B, hepatitis C, HIV and CMV DNA tests were negative. Urine and blood culture were negative. Echocardiography was performed, but revealed nothing specific and endocarditis was excluded. The empiric antibiotic therapy with clindamycin was started. Nuclear magnetic resonance (NMR) of the lumbar spine was firstly described as inconclusive, although the patient complained of constant pain aggravation. On the tenth day of hospitalization oedema with a tenderness of the left knee appeared. Due to exudates and excessive warmth of the left knee, arthrocentesis was performed. Sixty millilitres of turbid fluid was withdrawn. The synovial fluid was positive for Streptococcus sp. (saprophytic flora) thus a prolonged, targeted vancomycin therapy was started (the patient was allergic to penicillin). Gout was excluded. Moreover, because of the resistant pain and oedema of the left lower limb, Doppler ultrasound was performed and showed active deep vein thrombosis. Low molecular weight heparin therapy was administered in therapeutic dose. A control ultrasound examination revealed no features of thrombosis or valvular insufficiency. Despite antibiotic treatment further high inflammation parameters were still noted (CRP $149.4 \mathrm{mg} / \mathrm{l}$ ), thus further diagnosis for atypical infection was performed (the direct examination of synovial fluid for tuberculosis and in vitro QuantiFERON test, anti - Borrelia burgdorferi antibodies in class IgM and IgG - all negative). After failure of treatment and the patient's continuing complaints, the patient was referred to the Department of Rheumatology.

At the time of admission to the Department of Rheumatology the patient reported left knee pain sustained for more than a month and lumbar spine pain, severity independent of physical exercise, exacerbating during the night and accompanied by morning stiffness. Joint mobility within the normal range, soreness during loading and maximum limbs bent. In addition, Patrick sign was positive on the left side. After more detailed anamnesis the patient confirmed occurrence of typical inflammatory back pain of nearly 20 years. The pain typically worsened with inactivity and improved with exercise and non-steroid anti-inflammatory drugs (NSAI Ds) treatment. Morning stiffness was prolonged to nearly all day. Nocturnal pain was not awakening her. The patient's spine motion was markedly limited in all directions: Otto's test $2 \mathrm{~cm}$, Schober's test $1.5 \mathrm{~cm}$, chest expansion $3.5 \mathrm{~cm}$ deepening the cervical lordosis. Bath Ankylosing Spondylitis Disease Activity Index (BASDAI) was 6.2 whereas Bath Ankylosing Spondylitis Functional Index (BASFI) 2.5. There was neither edema nor mobility limitations in other peripheral joints. No other significant deviations from the norm in the rest of physical examination were noted. The symptoms were accompanied by high erythrocyte sedimentation rate (ESR) 98 $\mathrm{mm} / \mathrm{h}$, CRP $119.8 \mathrm{mg} / \mathrm{l}$ following an increase of creatinine concentration up to $144 \mu \mathrm{mol} / \mathrm{l}\left(\mathrm{GFR}_{\text {CKD-EPI }} 35.7 \mathrm{ml} /\right.$ $\min / 1.73 \mathrm{~m}^{2}$ ).

During hospitalization the antibiotic therapy started in the Department of Nephrology was completed, whilst the anti-coagulant therapy was continued. An x-ray of the lumbar spine and of the targeted sacroiliac joints revealed lesions characteristic of ankylosing spondylitis: vertebral body squaring, diffuse syndesmophyitic ankyloses giving a "bamboo spine" appearance and total ankyloses of sacroiliac joints (Fig. 1). The patient was HLA B27 positive, autoantibodies like the antinuclear antibodies (ANA), anti- cyclic citrullinated peptides antibodies and rheumatoid factor were negative. After the 

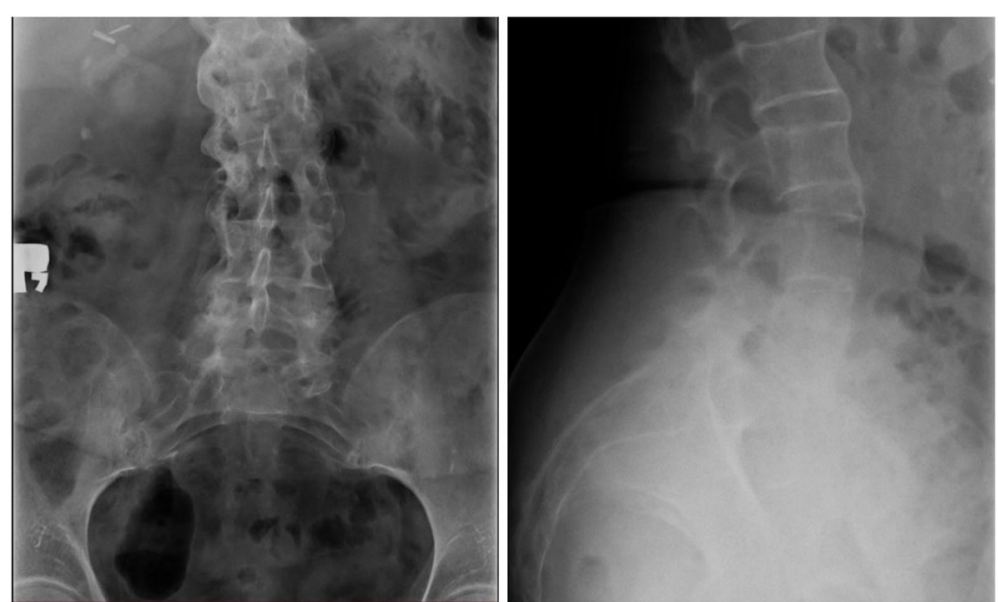

Fig. 1 X-ray revealed diffuse syndesmophyitic ankylosis at all levels from Th12 to L1 (also seen above Th12), calcifications of spinal ligaments, features of vertebral body squaring, radiological signs of spondylodiscitis at levels from $L 1$ to $L 4$ with the widening of intervertebral space, blurring the trabecular structure of L5 and L6 narrowing of intervertebral spaces L4-S1. Left site scoliosis

negative result of control culture of the synovial fluid, intrarticular injection of bethametazone and lignocaine mixture was administered. The fluid fulfilled the criteria for the inflammatory fluid type I (Table 1). Uric acid crystals were not detected. The NMR of the lumbar spine done in the Nephrology Unit was assessed once again by a more experienced radiologist in the Rheumatology Unit and was described as typical features of ankylosing spondylitis. A diagnosis of ankylosing spondylitis was posed (Tables 2 and 3) and the patient was started on oral sulphasalazine and NSAIDs. The treatment resulted in a significant decrease of CRP and improvement of the graft function ( $\mathrm{sCr} 92 \mu \mathrm{mol} / \mathrm{l}$, eGFR $61.4 \mathrm{ml} / \mathrm{min} / 1.73 \mathrm{~m}^{2}$ ). The diagnosis of chronic kidney disease (CKD) and KTx determined a reduction of sulphasalazine dose to $2 \times 500 \mathrm{mg}$ and after a short therapy the NSAIDs administration was reduced. Radiosynovectomy in the left knee joint was performed and after 2 weeks the patient, in good general condition, was discharged home.

Table 1 Synovial fluid parameters

\begin{tabular}{ll}
\hline Synovial fluid parameters & \\
\hline Volume & $60 \mathrm{ml}$ \\
Colour & Yellow \\
Clarity & Transparent \\
Viscosity & High \\
Total protein & $50.3 \mathrm{~g} / \mathrm{l}$ \\
Glucose level & Lower than blood \\
WBC & $800 / \mathrm{mm}^{3}$ \\
PMNs & $>50 \%$ \\
Culture & Negative \\
\hline
\end{tabular}

After 6 years of follow up during the routine visits in out-patient Transplantation Department the patient does not present any complaints associated with the left knee (no pain, tenderness, oedema). The graft function is still stable - creatinine concentration $90 \mu \mathrm{mol} / \mathrm{l}, \mathrm{GFR}_{\mathrm{CKD}-\mathrm{EPI}}$ $62 \mathrm{ml} / \mathrm{min} / 1.73 \mathrm{~m}^{2}$, she has been treated long-term with her base immunosuppressive therapy (stable dose of CsA $75 \mathrm{mg}$ bid, stable dose of MMF $500 \mathrm{mg}$ bid, and temporarily, increased steroid dose up to $20 \mathrm{mg}$ qd at the moment of AS diagnosis with the subsequent dose reduction to 5 $\mathrm{mg} \mathrm{qd})$, with reduced sulphasalazine dose $(2 \times 500 \mathrm{mg})$, with no adverse effects of such treatment.

\section{Discussion and conclusion}

Joint pain is a frequent problem in individuals with kidney disease and is common both before and after KTx [11]. However, kidney involvement can be one of the complications following rheumatic diseases and can occur as a secondary amyloidosis which is the most common cause of nephrotic syndrome in AS followed by IgA nephropathy, mesangioproliferative glomerulonephritis as well as, rarely, by membranous nephropathy or focal segmental glomerulosclerosis [10]. A study by Hill et al. showed an 18\% prevalence of CKD stages 3 to 5 in the rheumatic outpatient group, as compared to $5 \%$ reported within the general population [12]. On the other hand, it was shown that rheumatic diseases were the cause of ESRD and KTx in $14 \%$ of recipients transplanted in Poland between 1998 and 2015 years [13]. The authors showed that patients and graft survival were distinctly better in nonrheumatic recipients in comparison with rheumatic patients [13].

Differential diagnosis of joint and back pain in patients after successful KTx should take into account common complications of dialysis including crystal deposition disease, $\beta_{2}$ microglobulin amyloidosis, secondary hyperparathyroidism, 
Table 2 Amor diagnostic criteria for spondyloarthropathy

\begin{tabular}{lll}
\hline Amor Criteria & & Our patient \\
\hline Inflammatory back pain & 1 point & + \\
Unilateral buttock pain & 1 point & - \\
Alternating buttock pain & 2 point & - \\
Enthesitis & 2 point \\
Peripheral arthritis & 2 point \\
Dactylitis (sausage digit) & 2 point \\
Acute anterior uveitis & 2 point \\
HLA-B27 -positive or family history of spondyloarthropathy & 2 point \\
Good response to NSAIDs & 2 point \\
Diagnosis of spondyloarthropathy with 6 or more points & \\
\hline
\end{tabular}

or aluminium overload as well as articular complications followed by hepatitis $\mathrm{B}$ and $\mathrm{C}$ or a variety of other infections [14]. In KTx recipients some new musculoskeletal and articular disorders, like infection-related arthralgia, polyarticular gout, rheumatoid arthritis flare, or a medication-related adverse reaction, namely steroid induced osteopenia or osteoporosis, may occur [11].

To the best of our knowledge, the present work is the first case report of late onset of seronegative spondyloarthropathy in a transplanted patient despite ongoing immunosuppressive treatment. De novo seropositive erosive rheumatoid arthritis in a patient 7 years after KTx was described by Forslund et al. in 2005 [15].

Immunosuppressive treatment after transplantation on the one hand prevents rejection of the transplanted organ, and on the other hand is an excellent treatment for diseases of the autoimmune and inflammatory background, preventing an exaggerated immune response. However certain injuries may alter this balance leading to dysregulation of the intestinal immune environment promoting the development of chronic inflammatory disease [16]. Immunosuppression heightens susceptibility to infectious agents that may damage the epithelial barrier of the intestinal mucosa resulting in prolonged exposure to luminal antigens followed by chronic immune stimulation [16].

Calcineurin inhibitors including CsA and tacrolimus strongly inhibit production of interleukin-2 (IL-2), which is normally produced by $\mathrm{T}$ cells to induce expansion of antigen-specific clones in response to a previously encountered antigen. Deficiency of IL-2 can result in T-cell dysregulation, leading to the development of chronic inflammation [17]. Arthralgia, muscular side effects and osteoarthropathy are some of the described side effects of CsA associated therapy. It was shown that high CsA levels $(>200 \mathrm{ng} / \mathrm{ml}$ ) are the risk factors of joint pain in KTx recipients [11]. Our patient had the CsA trough level low, appropriate to time passed after KTx.

It has been found that mycophenolate mofetil can be associated with an acute inflammatory response characterized by fever, arthralgia, oligoarthritis and raised inflammatory markers appearing 3 to 5 days after initiation of therapy with MMF. Symptoms rapidly resolve with mycophenolate cessation. The pathogenesis has been associated with paradoxical pro-inflammatory reaction of polymorphonuclear neutrophils [18]. Our patient was treated with MMF for years, thus it is unlikely to have been the cause of symptoms.

The appearance of inflammatory disease regardless of adequate immunosuppressive therapy in transplant recipients is not unusual or impossible, however there is no data available associated with new onset of AS after transplantation. Moreover the most typical risk group of AS are young men, whereas the presented patient is a 58 year old woman. Very rarely does AS begin after the age of 40 resulting in difficulties in making the diagnosis,

Table 3 New York diagnostic criteria for spondyloarthropathy

\begin{tabular}{l}
\begin{tabular}{l} 
New York Criteria \\
\hline Low back pain with inflammatory characteristics \\
Limitation of lumbar spine motion in sagittal and frontal planes \\
Decreased chest expansion \\
Bilateral sacroiliitis grade 2 or higher \\
Unilateral sacroiliitis grade 3 or higher \\
Definite ankylosing spondylitis when the fourth or fifth criterion mentioned presents with any clinical criteria \\
\hline
\end{tabular}$+$ \\
\hline
\end{tabular}


since standard classification scales are not validated for patients above 45 years old [19, 20].

The diagnosis of arthritis is primarily clinical. The most typical clinical features of arthritis of native joints are acute joint pain, swelling, warmth, erythema, decreased range of motion, fever, and general malaise. AS is characterised by the presence of spinal pain, resulting in limitation of spinal mobility reported also by our patient. These symptoms can be supported by laboratory evidence like leukocytosis or inflammatory markers including CRP, procalcitonin and ESR, which can be useful especially in patients with native joints without underlying haematological or rheumatological conditions. However, all of these laboratory tests have quite low sensitivity and specificity. Laboratory tests are usually followed by X-ray, ultrasound, computer tomography (CT) or magnetic resonance imaging (MRI) of the involved joint. To diagnose joint infection aspiration of the joints can be performed, however mainly in large joints.

As written above the immunosuppressive treatment can be associated with increased risk of infectious diseases. Therefore, the most common cause of monoarticular arthritis in both immunocompetent and immunocompromised patients is septic arthritis [21]. Symptoms of AS (low back pain) were reported by our patient for 20 years, but as low back pain is a common condition and the patient did not present other features of spondyloarthropathy like enthesitis, dactylitis, uveitis, diarrhoea or family clustering, the patient was not referred to a rheumatologist and the complaints were described as non-specific, mechanical low back pain. It cannot be excluded that the symptoms of AS were present earlier but were masked by anti-inflammatory properties of immunosuppressive drugs.

Although most cases of viral and reactive arthritis are connected with infectious background, they are usually not categorized as septic arthritis [21]. Monoarticular arthritis can result from viral infection caused by parvovirus B19, HIV or rubella, as well as from the deposition of immune complexes and complement components in hepatitis B and C infections [19]. In our patient the viral infections were excluded.

Reactive arthritis usually arises from an acute infection of the genitourinary or gastrointestinal tract caused by Shigella spp., Salmonella spp., Campylobacter jejuni, or Yersinia enterocolitica. It may also be one of the manifestations of Lyme disease [21, 22].

In the traditional understanding of septic arthritis the most commonly implicated organisms causing bacteremic septic arthritis are Staphylococcus aureus, Streptococcus pyogenes, Neisseria meningitides, Neisseria gonorrhoeae, Klebsiella pneumonia, Escherichia coli, Proteus spp., Salmonella spp., Morganella morganii, Citrobacter spp., Serratias spp or Pseudomonas aerginosa [22-24]. Septic arthritis can also result from mycobacteria (Mycobacterium tuberculosis), or fungi infections (Histoplasma capsulatum, Blastomyces dermatitidis, Coccidioides immitis) [21].

The most common predisposing factors for septic arthritis include age greater than 80 years, diabetes mellitus, rheumatoid arthritis, prosthetic joint, recent joint surgery, skin infection and skin ulcers, intravenous drug abuse and alcoholism, and previous intra-articular corticosteroid administration [25].

Transplant patients are particularly susceptible to develop septic arthritis caused by unusual organisms. These infections are commonly disseminated, involving both the bones and joints [25]. In the first few weeks after transplantation, joint infections are often hematogenous and caused by health-care-associated pathogens such as Staphylococcus aureus (including MRSA) and gramnegative bacilli like Pseudomonas aeruginosa [26]. However, in the months following transplantation, as patients are maintained on immunosuppressive therapy, they are more prone to infections caused by fungi and mycobacteria [26]. In our recipients fungi and mycobacteria were excluded.

Gout etiology was thought unlikely in our patient as no crystals were present on joint aspirate and the patient had no history of gout, with only slight elevated serum uric acid concentration [19] - thus this diagnosis was excluded in our case.

Following the modified New York criteria for AS it has been shown that radiographic sacroilitis is a rather late finding in the disease course of many patients [3]. The most typical radiological evidence for AS are structural changes in the sacroiliac joints and the spine. In our patient the typical features like vertebral body squaring, diffuse syndesmophyitic ankyloses giving a "bamboo spine" appearance and total ankyloses of sacroiliac joints were described on X-ray, whereas MRI revealed nothing specific.

Progression of AS varies among individuals, but some general trends can be observed. Younger age ( $\leq 40$ years) at disease onset is usually associated with a predominance of axial symptoms, whereas patients with a later disease onset tend to present more peripheral manifestations [9]. Symptoms presented by our patient were associated with the spinal column despite her belonging to an older group.

The main purpose of treating the patient with AS is to improve long-term health-related quality of life by controlling the symptoms of the disease and averting continuous structural damage, thus leading to better joint function followed by improved social participation [3]. The treatment of AS should be personalised according to the present symptoms of the disease, patient's comorbidities, possible complications resulting from the treatment and psychosocial factors [3]. 
The first-line drug treatment for patients complaining of pain and stiffness are NSAIDs administered up to the maximum dose, taking into consideration risks and benefits resulting from the treatment [27, 28]. Data from some studies revealed that in young patients failure to administered NSAIDs is associated with an increased mortality $[29,30]$. For patients with good response to NSAIDs continuous use is recommended [3]. This firstline treatment should be enhanced by physical therapy including home exercises and by smoking cessation [31]. In patients who are contraindicated to NSAIDs or poorly tolerate this treatment, analgesics such as paracetamol and opioid-(like) drugs should be considered [3].

Lack of efficacy or presence of toxicity with NSAIDs is an indication to consider alternative treatments either with glucocorticoid, sulfasalazine or biological diseasemodifying antirheumatic drugs (bDMARDs) [3].

Steroid injections directed to the local site of musculoskeletal inflammation may be recommended in patients with predominant peripheral manifestation as an option to treat arthritis and enthesitis, although direct evidence is lacking. Patients with axial disease should not be treated with systemic glucocorticoids for a long time [3].

Sulfasalazine may be considered in patients with peripheral arthritis, however it does not alleviate axial symptoms [32].

High disease activity defined as BASDAI $\geq 4$ despite conventional treatment, elevated CRP, presence of inflammation on MRI of the SI joints and/or spine or presence of radiographic sacroiliitis is an indication to start bDMARDs and current practice is to start with tumour necrosis factor inhibitor (TNFi) therapy [31].

Our patient due to KTx was put on immunosuppressive therapy including CsA, MMF and steroids. Given the ongoing severity of the patient's symptoms, no response to maintenance immunosuppressive therapy, contraindication to NSAIDs, a change in management was instituted by the administration of sulfasalazine. Improvement in the patient's inflammatory markers and arthritis was noticed with subsidence of pain complaints and normalization of CRP within 2 weeks. She has had no further recurrence of acute inflammatory arthritis.

The establishing of proper diagnosis and management of a patient after KTx is difficult. Despite long-term immunosuppressive treatment in solid organ transplant recipients, in the differential diagnosis of joint pain the development of de novo arthritis should be considered, and when conclusive diagnosis is made, the patient may require administration of additional immunosuppressive drugs.

\section{Abbreviations}

ANA: Antinuclear antibodies; anti-CCP: Anti- cyclic citrullinated peptides; AS: Ankylosing spondylitis; BASDAl: Bath Ankylosing Spondylitis Disease Activity Index; BASFI: Bath Ankylosing Spondylitis Functional Index; bDMARDs: Biological disease-modifying antirheumatic drugs; CKD: Chronic kidney disease; CRP: C-reactive protein; CSA: Cyclosporine A; CT: Computer tomography; eGFR: Estimated glomerular filtration rate; ESR: Erythrocyte sedimentation rate; ESRD: End-stage renal disease; HPF: Higher-power field; Ktx: Kidney transplantation; MMF: Mycophenolate mofetil; MRI: Magnetic resonance imaging; NMR: Nuclear magnetic resonance; NSAIDs: Non-steroid anti-inflammatory drugs; SCr: Serum creatinine concentration; TNFi: Tumour necrosis factor inhibitor; WBC: White blood count

\section{Acknowledgements}

Not applicable.

\section{Authors' contributions}

All authors have read and have approved the submitted version of the manuscript. AZB: designed the case report plan, described the subject medical history and was the primary author for the manuscript; OB: helped design the case report plan, IK helped in writing the manuscript, JM helped in writing the manuscript. All authors have agreed both to be personally accountable for the author's own contributions and to ensure that questions related to the accuracy or integrity of any part of the work, even ones in which the author was not personally involved, are appropriately investigated, resolved, and the resolution documented in the literature.

Funding

Not applicable.

\section{Availability of data and materials}

The patient results used and/or analysed during the current case report are available from the corresponding author on reasonable request.

Ethics approval and consent to participate

Not applicable (case report).

\section{Consent for publication}

The patient in written consent agreed to publish the laboratory as well as radiological results.

\section{Competing interests}

None of the authors claims a conflict of interest. All authors declare no financial and non-financial competing interests.

\section{Author details}

'Department of Internal Diseases and Transplant Nephrology, The Medical University of Lodz, Lodz, Poland. ' Department of Rheumatology, The Medical University of Lodz, Lodz, Poland.

Received: 9 October 2020 Accepted: 21 January 2021

Published online: 31 January 2021

References

1. Asif A, Toral C, Diego J, Miller J, Roth D. De novo ANCA-associated vasculitis occurring 14 years after kidney transplantation. Am J Kidney Dis. 2000;35(3): E10.

2. Moran S, Little MA. Renal transplantation in antineutrophil cytoplasmic antibody-associated vasculitis. Curr Opin Rheumatol. 2014;26:37-41.

3. Van der Heljde D, Ramiro S, Landewe R, et al. 2016 update of the ASASEULAR management recommendations for axial spondyloarthritis. Ann Rheum Dis. 2017;76:978-91.

4. Terenzi R, Monti S, Tesei G, Carli L. One year in review 2017: spondyloarthritis. Clin Exp Rheumatol. 2018;36:1-14.

5. Dougados M, Baeten D. Spondyloarthritis. Lancet. 2011;377:2127-37.

6. Davatchi F, Sandoughi M, Moghimi N, Jamshidi AR, Tehrani Banihashemi A, Zakeri Z, Sadeghi AB. Epidemiology of rheumatic diseases in Iran from analysis of four COPCORD studies. Int J Rheum Dis. 2016;19:1056-62.

7. Dagfinrud H, Mengshoel AM, Hagen KB, Loge JH, Kvien TK. Health status of patients with ankylosing spondylitis: a comparison with the general population. Ann Rheum Dis. 2004;63:1605-10.

8. Stolwijk C, van Tubergen A, Castillo-Ortiz JD, Boonen A. Prevalence of extraarticular manifestations in patients with ankylosing spondylitis: a systematic review and meta-analysis. Ann Rheum Dis. 2015;74:65-73.

9. Strand V, Singh JA. Patient burden of axial Spondyloarthritis. J Clin Rheumatol. 2017;23:383-91. 
10. Mercieca C, van der Horst-Bruinsma IE, Borg AA. Pulmonary, renal and neurological comorbidities in patients with ankylosing spondylitis; implications for clinical practice. Curr Rheumatol Rep. 2014;16:434.

11. Kart-Köseoglu H, Yücel AE, Isiklar I, Türker I, Akçali Z, Haberal M. Joint pain and arthritis in renal transplant recipients and correlation with cyclosporine therapy. Rheumatol Int. 2003;23:159-62.

12. Hill AJ, Thomson RJ, Hunter JA, Traynor JP. The prevalence of chronic kidney disease in rheumatology outpatients. Scott Med J. 2009;54:9e12.

13. Ciszek M, Kisiel B, Czerwinski J, Hryniewiecka E, Lewandowska D, Borczon S, Tlustochowicz W, Paczek L. Kidney transplant recipients with rheumatic diseases: epidemiological data from the polish transplant registries 19982015. Transplant Proc. 2018;50:1654-7.

14. Kessler BM, Netter P, Azoulay E, Mayeux D, Pere P, Gaucher A. Dialysisassociated arthropathy: a multicentre survey of 171 patients receiving haemodialysis for over 10 years. Br J Rheumatol. 1992:31:157-62.

15. Forslund T, Ahonen J, Elomaa E, Parviainen T, Matinlauri I. De novo seropositive rheumatoid arthritis during immunosuppressive treatment after kidney transplantation. Clin Nephrol. 2005;64:231-5.

16. Pittman ME, Jessurun J, Yantiss RK. Differentiating Posttransplant inflammatory bowel disease and other Colitides in renal transplant patients. Am J Surg Pathol. 2017;41:1666-74.

17. Indriolo A, Ravelli P. Clinical management of inflammatory bowel disease in the organ recipient. World J Gastroenterol. 2014;20:3525-33.

18. Hochegger K, Gruber J, Lhotta K. Acute inflammatory syndrome induced by mycophenolate mofetil in a patient following kidney transplantation. Am J Transplant. 2006:6:852-4

19. Braun J, Sieper J. Ankylosing spondylitis. Lancet. 2007;369:1379-90.

20. Rudwaleit M, Khan MA, Sieper J. The challenge of diagnosis and classification in early ankylosing spondylitis: do we need new criteria? Arthritis Rheum. 2005;52:1000-8.

21. Wang DA, Tambyah PA. Septic arthritis in immunocompetent and immunosuppressed hosts. Best Pract Res Clin Rheumatol. 2015;29:275-89.

22. Murillo O, Grau I, Lora-Tamayo J, Gomez-Junyent J, Ribera A, Tubau F. The changing epidemiology of bacteraemic osteoarticular infections in the early 21st century. Clin Microbiol Infect. 2015;21:254.e1-8.

23. Zhu Z, Kang Y, Lin Z, Huang Y, Lv H, Li Y. X-linked agammaglobulinemia combined with juvenile idiopathic arthritis and invasive Klebsiella pneumoniae polyarticular septic arthritis. Clin Rheumatol. 2015;34:397-401.

24. Vardakas KZ, Kontopidis I, Gkegkes ID, Rafailidis PI, Falagas ME. Incidence, characteristics, and outcomes of patients with bone and joint infections due to community-associated methicillin-resistant Staphylococcus aureus: a systematic review. Eur J Clin Microbiol Infect Dis. 2013;32(6):711-21.

25. Margaretten ME, Kohlwes J, Moore D, Bent S. Does this adult patient have septic arthritis? J Am Med Assoc. 2007;297(13):1478-88.

26. Kim HK, Park YK, Wang HJ, Kim BW, Shin SY, Lim S-K, et al. Epidemiology and clinical features of post-transplant bloodstream infection: an analysis of 222 consecutive liver transplant recipients. Infect Chemother. 2013;45:315-24.

27. Sieper J, Lenaerts J, Wollenhaupt J, Rudwaleit M, Mazurov VI, Myasoutova L, et al. Efficacy and safety of infliximab plus naproxen versus naproxen alone in patients with early, active axial spondyloarthritis: results from the double-blind, placebo-controlled INFAST study, part 1. Ann Rheum Dis. 2014;73:101-7.

28. Kroon F, Landewé R, Dougados M, van der Heijde D. Continuous NSAID use reverts the effects of inflammation on radiographic progression in patients with ankylosing spondylitis. Ann Rheum Dis. 2012;71:1623-9.

29. Bakland G, Gran JT, Nossent JC. Increased mortality in ankylosing spondylitis is related to disease activity. Ann Rheum Dis. 2011;70:1921-5.

30. Haroon NN, Paterson JM, Li P, Inman RD, Haroon N. Patients with ankylosing spondylitis have increased cardiovascular and cerebrovascular mortality: a population-based study. Ann Intern Med. 2015;163:409-16.

31. Chung HY, Machado P, van der Heijde D, D'Agostino MA, Dougados M. Smokers in early axial spondyloarthritis have earlier disease onset, more disease activity, inflammation and damage, and poorer function and healthrelated quality of life: results from the DESIR cohort. Ann Rheum Dis. 2012; 71:809-16.

32. Chen J, Liu C. Sulfasalazine for ankylosing spondylitis. Cochrane Database Syst Rev. 2005;2:CD004800.

\section{Publisher's Note}

Springer Nature remains neutral with regard to jurisdictional claims in published maps and institutional affiliations.

\section{Ready to submit your research? Choose BMC and benefit from:}

- fast, convenient online submission

- thorough peer review by experienced researchers in your field

- rapid publication on acceptance

- support for research data, including large and complex data types

- gold Open Access which fosters wider collaboration and increased citations

- maximum visibility for your research: over $100 \mathrm{M}$ website views per year

At $\mathrm{BMC}$, research is always in progress.

Learn more biomedcentral.com/submissions 